PROCEEDINGS OF THE

AMERICAN MATHEMATICAL SOCIETY

Volume 140, Number 7, July 2012, Pages 2453-2463

S 0002-9939(2011)11181-X

Article electronically published on November 21, 2011

\title{
MEAN VALUE PROPERTY FOR $p$-HARMONIC FUNCTIONS
}

\author{
TIZIANA GIORGI AND ROBERT SMITS
}

(Communicated by Matthew J. Gursky)

\begin{abstract}
We derive a mean value property for $p$-harmonic functions in two dimensions, $1<p<\infty$, which holds asymptotically in the viscosity sense. The formula coincides with the classical mean value property for harmonic functions, when $p=2$, and is a consequence of a representation for the Game $p$-Laplacian obtained via $p$-averaging.
\end{abstract}

\section{INTRODUCTION}

A recent article by Manfredi et al. [6] (see also [9]) characterizes $p$-harmonic functions via a weak asymptotic formula which holds in a suitably defined viscosity sense. Inspired by their results and by our recent work 4, where we present a numerical algorithm for the Game $p$-Laplace operator based on the idea of $p$-average, we derive a generalization in a viscosity sense to two-dimensional $p$-harmonic functions, $1<p<\infty$, of the classical mean value property for harmonic functions.

The variational $p$-Laplace operator is defined, for $1<p<\infty$, as

$$
\Delta_{p} u \equiv \operatorname{div}\left(|\nabla u|^{p-2} \nabla u\right),
$$

while the Game $p$-Laplacian, recently introduced in [7 to model a stochastic game called Tug of war with noise, reads as

$$
\Delta_{p}^{G} u \equiv \frac{1}{p}|\nabla u|^{2-p} \operatorname{div}\left(|\nabla u|^{p-2} \nabla u\right) .
$$

A function $u \in C^{0}(\Omega)$, with $\Omega \subset \mathcal{R}^{2}$ a smooth domain, is called $p$-harmonic in $\Omega$ if it is a viscosity solution of $\Delta_{p} u=0$ (see Definition 2.1).

The focus of this paper is in providing a representation of $p$-harmonic functions that for the case $p=2$ reproduces the mean value property. Nevertheless, it will be clear that our main interest is the Game $p$-Laplacian and that our approach sheds light on the local properties of the solution of the Game $p$-Laplace operator. The representation formula derived was suggested to us by the numerical approximation we propose in an upcoming paper 4. An insight on the local properties of the Game $p$-Laplacian suggests that the value of a solution at a given point is related to the $p$-average on small balls centered at that point. The numerical solution that we construct, in the case of dimension $n=2$, using this idea satisfies a discrete analogue of our proposed generalized mean value formula.

We derive the following main results. Our first theorem finds an expansion for $C^{2}$ functions in terms of the Game $p$-Laplacian:

Received by the editors November 1, 2010 and, in revised form, February 26, 2011.

2010 Mathematics Subject Classification. Primary 35J92, 35D40, 35J60, 35J70.

Funding for the first author was provided by National Science Foundation Grant \#DMS0604843.

(C)2011 American Mathematical Society 2453

Reverts to public domain 28 years from publication 
Theorem 1.1. Let $\phi \in C^{2}(\Omega)$, where $\Omega \subset \mathcal{R}^{2}$ is a smooth domain, and let $x_{0} \in \Omega$. If $\nabla \phi\left(x_{0}\right) \neq 0$, then for any $\epsilon>0$ such that $B_{\epsilon}\left(x_{0}\right) \subset \Omega$ we have

$$
\phi\left(x_{0}\right)=\frac{\int_{B_{\epsilon}\left(x_{0}\right)}\left|\nabla \phi\left(x_{0}\right) \cdot\left(x-x_{0}\right)\right|^{p-2} \phi(x) d x}{\int_{B_{\epsilon}\left(x_{0}\right)}\left|\nabla \phi\left(x_{0}\right) \cdot\left(x-x_{0}\right)\right|^{p-2} d x}-\frac{p}{p+2} \frac{\epsilon^{2}}{2} \Delta_{p}^{G} \phi\left(x_{0}\right)+o\left(\epsilon^{2}\right) .
$$

Here $B_{\epsilon}\left(x_{0}\right)$ denotes the ball of radius $\epsilon$ and center $x_{0}$.

We then use this representation to derive our weak mean value formula for $p$-harmonic functions.

Theorem 1.2. Let $u$ be a continuous function in $\Omega \subset \mathcal{R}^{2}$, and let $x_{0} \in \Omega$. For any $\epsilon>0$ such that $B_{\epsilon}\left(x_{0}\right) \subset \Omega$ we have that

$$
u\left(x_{0}\right)=\frac{\int_{B_{\epsilon}\left(x_{0}\right)}\left|\nabla u\left(x_{0}\right) \cdot\left(x-x_{0}\right)\right|^{p-2} u(x) d x}{\int_{B_{\epsilon}\left(x_{0}\right)}\left|\nabla u\left(x_{0}\right) \cdot\left(x-x_{0}\right)\right|^{p-2} d x}+o\left(\epsilon^{2}\right)
$$

holds in the viscosity sense if and only if $u$ is p-harmonic; that is, $u$ is a viscosity solution of

$$
\Delta_{p} u\left(x_{0}\right)=0 .
$$

We present detailed proofs for the case of smooth domains $\Omega \subset \mathcal{R}^{2}$, but from our treatment it will be clear how to obtain generalizations to dimensions $n>2$.

The paper is organized as follows. In Section 2 we recall some definitions and background results. In Section 3 we derive the representation formula for $C^{2}$ functions and discuss why this is the correct local way of describing $p$-harmonic functions. In Section 4 we prove Theorem 1.2. To conclude, in Section 5 we derive a similar result for the non-homogeneous Game $p$-Laplacian.

\section{2. $p$-Laplacian and Game $p$-Laplacian}

Our representation formula for smooth functions is based on the so-called Game $p$-Laplacian introduced by Peres and Sheffield 7, and its proof is based on the characterization of $\Delta_{p}^{G}$ as a convex combination of two limiting operators.

When $p=\infty$, traditionally the $\infty$-Laplacian is given by

$$
\Delta_{\infty} u \equiv \sum_{i, j} \frac{\partial u}{\partial x_{i}} \frac{\partial u}{\partial x_{j}} \frac{\partial^{2} u}{\partial x_{i} \partial x_{j}}
$$

while the Game $\infty$-Laplacian is its 1-homogeneous renormalized version:

$$
\Delta_{\infty}^{G} u \equiv|\nabla u|^{-2} \sum_{i, j} \frac{\partial u}{\partial x_{i}} \frac{\partial u}{\partial x_{j}} \frac{\partial^{2} u}{\partial x_{i} \partial x_{j}} .
$$

For $p=1$, we can set $p=1$ in (1) and obtain

$$
\Delta_{1} u \equiv \operatorname{div}\left(|\nabla u|^{-1} \nabla u\right),
$$

while for the Game 1-Laplacian we follow [7] and define it in terms of the Laplace operator and the Game $\infty$-Laplacian:

$$
\Delta_{1}^{G} u \equiv \Delta_{2} u-\Delta_{\infty}^{G} u .
$$

If $u$ is a smooth function, by expanding the derivatives, one obtains

$$
\Delta_{p}^{G} u=\frac{1}{p} \Delta_{2} u+\frac{p-2}{p}|\nabla u|^{-2} \sum_{i, j} \frac{\partial u}{\partial x_{i}} \frac{\partial u}{\partial x_{j}} \frac{\partial^{2} u}{\partial x_{i} \partial x_{j}},
$$


which allows us to think of $\Delta_{p}^{G}$ as the convex combination of the two limiting cases, that is,

$$
\Delta_{p}^{G}=\frac{1}{p} \Delta_{1}^{G}+\frac{1}{q} \Delta_{\infty}^{G},
$$

with $q$ the conjugate exponent of $p$. Furthermore, the Game 1-Laplacian and the Game $p$-Laplacian for $\nabla u \neq 0$ can then be rewritten as the second derivative in the orthogonal direction of $\nabla u$ and in the direction of $\nabla u$, respectively. That is,

$$
\Delta_{1}^{G} u=\left|\nabla u^{\perp}\right|^{-2}\left\langle D^{2} u \nabla u^{\perp}, \nabla u^{\perp}\right\rangle \text {, }
$$

and

$$
\Delta_{\infty}^{G} u=|\nabla u|^{-2}\left\langle D^{2} u \nabla u, \nabla u\right\rangle
$$

where $D^{2} u$ denotes the Hessian matrix.

In the homogeneous case, solutions to the Game $p$-Laplacian agree with the ones of the $p$-Laplacian. Also note that the Game $\infty$-Laplacian is the limit as $p \rightarrow \infty$ of the Game $p$-Laplacian, a fact which is not true for the $p$-Laplacian.

The fundamental difference between the classical $p$-Laplacian and the Game $p$-Laplacian is that the former can be obtained as the Euler-Lagrange equation of an energy functional. Additionally, while for $1<p<\infty$ both operators are degenerate, for $\nabla u=0$ the Game $p$-Laplacian and the 1-Laplacian are singular, so suitable definitions of viscosity solutions need to be given.

We recall that Juutinen and the coauthors in [5] show that for the $p$-Laplacian when $1<p<\infty$ the notions of viscosity solution and weak solution are equivalent. Therefore, we will work with viscosity solutions for both operators.

We consider the definition of viscosity solution for the $p$-Laplacian provided in 6]:

Definition 2.1. Let $\Omega \subset \mathcal{R}^{2}$ be a smooth domain, and $1<p<\infty$. Then:

(i) We say that an upper semi-continuous function $u$ is a viscosity subsolution of $\Delta_{p} u=0$ in $\Omega$ if for any $\phi \in C^{2}$ such that $u-\phi$ has a strict local maximum at $x \in \Omega$, we have

$$
-(p-2) \Delta_{\infty}^{G} \phi(x)-\Delta_{2} \phi(x) \leq 0 \text { whenever } \nabla \phi(x) \neq 0 .
$$

(ii) We say that a lower semi-continuous function $u$ is a viscosity supersolution of $\Delta_{p} u=0$ in $\Omega$ if for any $\phi \in C^{2}$ such that $u-\phi$ has a strict local minimum at $x \in \Omega$, we have

$$
-(p-2) \Delta_{\infty}^{G} \phi(x)-\Delta_{2} \phi(x) \geq 0 \text { whenever } \nabla \phi(x) \neq 0 .
$$

(iii) If $u$ is both a subsolution and a supersolution everywhere in $\Omega$, we say that $u$ is a viscosity solution of $\Delta_{p} u=0$ in $\Omega$.

Various equivalent definitions of viscosity solutions for the Game $p$-Laplacian operator can be given and are found in the literature. The most suitable for our treatment is the one obtained by following the definition in the classical paper of Barles and Souganidis [1].

Definition 2.2. Consider a smooth domain $\Omega \subset \mathcal{R}^{2}$, and let $1<p<\infty$. If $f$ is a continuous function, we say that an upper semi-continuous function $u$ (respectively, lower semi-continuous) is a viscosity subsolution (respectively, supersolution) of

$$
-\Delta_{p}^{G} u=f(x) \text { in } \Omega
$$


if for any $\phi \in C^{2}$ such that $u-\phi$ has a local maximum (respectively, local minimum) at $x \in \Omega$, we have:

(i) $-\Delta_{p}^{G} \phi(x) \leq f(x) \quad$ if $\nabla \phi(x) \neq 0 \quad$ (respectively, $\left.-\Delta_{p}^{G} \phi(x) \geq f(x)\right)$;

(ii) $-\frac{\lambda_{1}}{p}-\frac{\lambda_{2}}{q} \leq f(x)$ if $\nabla \phi(x)=0$ and $p \geq 2$

(respectively, $\left.-\frac{\lambda_{1}}{q}-\frac{\lambda_{2}}{p} \geq f(x)\right)$;

$-\frac{\lambda_{1}}{q}-\frac{\lambda_{2}}{p} \leq f(x)$ if $\nabla \phi(x)=0$ and $1<p<2$

(respectively, $-\frac{\lambda_{1}}{p}-\frac{\lambda_{2}}{q} \geq f(x)$ );

here $\frac{1}{p}+\frac{1}{q}=1$, and $\lambda_{1} \leq \lambda_{2}$ are the eigenvalues of $D^{2} \phi(x)$.

Remark 2.1. Part (ii) of the definition of viscosity subsolution (supersolution) is implied by the condition

$(\text { ii })^{\prime}-\Delta_{2}^{G} \phi(x) \leq f(x)$ whenever $\nabla \phi(x)=0$ (respectively, $\left.-\Delta_{2}^{G} \phi(x) \geq f(x)\right)$.

This is a consequence of the fact that

$$
-\frac{1}{p} \lambda_{1}-\frac{1}{q} \lambda_{2} \leq-\Delta_{2}^{G} \phi(x) \leq-\frac{1}{q} \lambda_{1}-\frac{1}{p} \lambda_{2} \text {, if } p \geq 2,
$$

and

$$
-\frac{1}{q} \lambda_{1}-\frac{1}{p} \lambda_{2} \leq-\Delta_{2}^{G} \phi(x) \leq-\frac{1}{p} \lambda_{1}-\frac{1}{q} \lambda_{2}, \text { if } 1<p<2 .
$$

Recall that $\Delta_{2}^{G} \phi=\frac{1}{2} \Delta_{2} \phi$.

Uniqueness for viscosity solutions of non-linear operators that are singular at isolated points typically does not depend on the particular value one assigns to these points as long as this is chosen in a consistent manner (see for example Section 9 in [3]). Additionally, our numerical results in 4 show that the numerical approximation converges to solutions that verify (ii)'. Therefore, we will use the following definition for a viscosity solution of the Game $p$-Laplacian:

Definition 2.3. We say that a function $u$ is a viscosity solution of $-\Delta_{p}^{G} u=f$ for $1<p<\infty$ if $u$ is a subsolution and supersolution according to (ii) of Definition 2.2 and (ii)' in Remark 2.1.

\section{Representation FORMUla}

Theorem 3.1. Let $\Omega \subset \mathcal{R}^{2}$ be a smooth domain. Given $\phi \in C^{2}(\Omega)$ and $x_{0} \in \Omega$ for which $\nabla \phi\left(x_{0}\right) \neq 0$, we have that for any $\epsilon>0$ such that $B_{\epsilon}\left(x_{0}\right) \subset \Omega$ it holds that

$$
\phi\left(x_{0}\right)=\frac{\int_{B_{\epsilon}\left(x_{0}\right)}\left|\nabla \phi\left(x_{0}\right) \cdot\left(x-x_{0}\right)\right|^{p-2} \phi(x) d x}{\int_{B_{\epsilon}\left(x_{0}\right)}\left|\nabla \phi\left(x_{0}\right) \cdot\left(x-x_{0}\right)\right|^{p-2} d x}-\frac{p}{p+2} \frac{\epsilon^{2}}{2} \Delta_{p}^{G} \phi\left(x_{0}\right)+o\left(\epsilon^{2}\right) .
$$

Here $B_{\epsilon}\left(x_{0}\right)$ denotes the ball of radius $\epsilon$ and center $x_{0}$.

Proof. Take $x=\left(x_{1}, x_{2}\right) \in \mathcal{R}^{2}$ and denote by $\mathbf{e}_{1}=(1,0)$ the unit director of the $x_{1}$-axis. Assume $\phi \in C^{2}(\Omega), x_{0} \in \Omega$ and $\nabla \phi\left(x_{0}\right) \neq 0$. Without loss of generality, we can assume $x_{0}=0$ and $\nabla \phi\left(x_{0}\right)=\left|\nabla \phi\left(x_{0}\right)\right| \mathbf{e}_{1}$. Equation (므) then gives

$$
\Delta_{\infty}^{G} \phi(0)=\partial_{11} \phi(0)
$$


while (5) yields

$$
\Delta_{1}^{G} \phi(0)=\partial_{22} \phi(0)
$$

For any $\epsilon>0$ such that $B_{\epsilon} \equiv B_{\epsilon}(0) \subset \Omega$, if $1<p<\infty$, we integrate over $B_{\epsilon}$ to obtain

$$
\begin{aligned}
& \int_{B_{\epsilon}}|\nabla \phi(0) \cdot x|^{p-2}\left\langle D^{2} \phi(0) x, x\right\rangle d x \\
& =|\nabla \phi(0)|^{p-2} \int_{B_{\epsilon}}\left|x_{1}\right|^{p-2}\left\langle D^{2} \phi(0) x, x\right\rangle d x \\
& =2|\nabla \phi(0)|^{p-2} \int_{B_{\epsilon} \cap\left\{x_{1}>0\right\}} x_{1}^{p-2}\left\langle D^{2} \phi(0) x, x\right\rangle d x,
\end{aligned}
$$

which by (90) and (10) implies

$$
\begin{aligned}
& \int_{B_{\epsilon}}|\nabla \phi(0) \cdot x|^{p-2}\left\langle D^{2} \phi(0) x, x\right\rangle d x \\
& =\quad 2|\nabla \phi(0)|^{p-2}\left[\Delta_{\infty}^{G} \phi(0) \int_{B_{\epsilon} \cap\left\{x_{1}>0\right\}} x_{1}^{p} d x\right. \\
& +2 \partial_{12} \phi(0) \int_{B_{\epsilon} \cap\left\{x_{1}>0\right\}} x_{1}^{p-2} x_{1} x_{2} d x \\
& \left.\quad+\Delta_{1}^{G} \phi(0) \int_{B_{\epsilon} \cap\left\{x_{1}>0\right\}} x_{1}^{p-2} x_{2}^{2} d x\right] .
\end{aligned}
$$

Also, note that

$$
\int_{B_{\epsilon}}|\nabla \phi(0) \cdot x|^{p-2} d x=2|\nabla \phi(0)|^{p-2} \int_{B_{\epsilon} \cap\left\{x_{1}>0\right\}} x_{1}^{p-2} d x .
$$

To compute the integrals in (11) and (12), we use polar coordinates and derive

$$
\int_{B_{\epsilon} \cap\left\{x_{1}>0\right\}} x_{1}^{p} d x=\int_{0}^{\epsilon} \int_{-\frac{\pi}{2}}^{\frac{\pi}{2}}(r \cos \theta)^{p} d \theta r d r=\frac{\epsilon^{p+2}}{p+2} \int_{-\frac{\pi}{2}}^{\frac{\pi}{2}}(\cos \theta)^{p} d \theta,
$$

as well as

$$
\begin{aligned}
& \int_{B_{\epsilon} \cap\left\{x_{1}>0\right\}} x_{1}^{p-2} x_{2}^{2} d x=\int_{0}^{\epsilon} \int_{-\frac{\pi}{2}}^{\frac{\pi}{2}}(r \cos \theta)^{p-2}(r \sin \theta)^{2} d \theta r d r \\
& =\frac{\epsilon^{p+2}}{p+2} \int_{-\frac{\pi}{2}}^{\frac{\pi}{2}}(\cos \theta)^{p-2}\left(1-\cos ^{2} \theta\right) d \theta \\
& =\frac{\epsilon^{p+2}}{p+2}\left[\int_{-\frac{\pi}{2}}^{\frac{\pi}{2}}(\cos \theta)^{p-2} d \theta-\int_{-\frac{\pi}{2}}^{\frac{\pi}{2}}(\cos \theta)^{p} d \theta\right]
\end{aligned}
$$

while by symmetry we see that

$$
\begin{aligned}
& \int_{B_{\epsilon} \cap\left\{x_{1}>0\right\}} x_{1}^{p-2} x_{1} x_{2} d x=\int_{0}^{\epsilon} \int_{-\frac{\pi}{2}}^{\frac{\pi}{2}}(r \cos \theta)^{p-1}(r \sin \theta) d \theta r d r \\
& =\frac{\epsilon^{p+2}}{p+2} \int_{-\frac{\pi}{2}}^{\frac{\pi}{2}}(\cos \theta)^{p-1} \sin \theta d \theta=0 .
\end{aligned}
$$


For the last integral that we need, we find that

$$
\int_{B_{\epsilon} \cap\left\{x_{1}>0\right\}} x_{1}^{p-2} d x=\int_{0}^{\epsilon} \int_{-\frac{\pi}{2}}^{\frac{\pi}{2}}(r \cos \theta)^{p-2} d \theta r d r=\frac{\epsilon^{p}}{p} \int_{-\frac{\pi}{2}}^{\frac{\pi}{2}}(\cos \theta)^{p-2} d \theta .
$$

We next substitute the above integrals in (11) and (12) to gather

$$
\frac{\int_{B_{\epsilon}}|\nabla \phi(0) \cdot x|^{p-2}\left\langle D^{2} \phi(0) x, x\right\rangle d x}{\int_{B_{\epsilon}}|\nabla \phi(0) \cdot x|^{p-2} d x}=\epsilon^{2} \frac{p}{p+2} \Delta_{p}^{G} \phi(0),
$$

where we used the elementary equality

$$
\frac{\int_{-\frac{\pi}{2}}^{\frac{\pi}{2}}(\cos \theta)^{p} d \theta}{\int_{-\frac{\pi}{2}}^{\frac{\pi}{2}}(\cos \theta)^{p-2} d \theta}=\frac{1}{q} \quad \text { for any } 1<p<\infty, \text { and } \frac{1}{p}+\frac{1}{q}=1 .
$$

However, now the theorem is a consequence of the Taylor expansion. In fact, given $x \in B_{\epsilon}$, since $\phi \in C^{2}$, we know that

$$
\phi(x)=\phi(0)+\nabla \phi(0) \cdot x+\frac{1}{2}\left\langle D^{2} \phi(0) x, x\right\rangle+o\left(|x|^{2}\right),
$$

as $|x| \rightarrow 0$. Therefore,

$$
\begin{aligned}
& \int_{B_{\epsilon}}|\nabla \phi(0) \cdot x|^{p-2} \phi(x) d x=\phi(0) \int_{B_{\epsilon}}|\nabla \phi(0) \cdot x|^{p-2} d x \\
& +\frac{1}{2} \int_{B_{\epsilon}}|\nabla \phi(0) \cdot x|^{p-2}\left\langle D^{2} \phi(0) x, x\right\rangle d x+o\left(\epsilon^{2+p}\right),
\end{aligned}
$$

since by symmetry one has

$$
\int_{B_{\epsilon}}|\nabla \phi(0) \cdot x|^{p-2} \nabla \phi(0) \cdot x d x=0,
$$

and, using (12) and (13), we find

$$
\begin{aligned}
& 0 \leq\left.\frac{1}{\epsilon^{2+p}}\left|\int_{B_{\epsilon}}\right| \nabla \phi(0) \cdot x\right|^{p-2} o\left(|x|^{2}\right) d x \mid \\
& \leq \frac{\left|o\left(\epsilon^{2}\right)\right|}{\epsilon^{2}} \frac{1}{p} \int_{-\frac{\pi}{2}}^{\frac{\pi}{2}}(\cos \theta)^{p} d \theta \rightarrow 0 \quad \text { as } \epsilon \rightarrow 0 .
\end{aligned}
$$

Dividing by the coefficient of $\phi(0)$ in (15) and by using (14) and (16), we obtain

$$
\phi(0)=\frac{\int_{B_{\epsilon}}|\nabla \phi(0) \cdot x|^{p-2} \phi(x) d x}{\int_{B_{\epsilon}}|\nabla \phi(0) \cdot x|^{p-2} d x}-\frac{\epsilon^{2}}{2} \frac{p}{p+2} \Delta_{p}^{G} \phi(0)+o\left(\epsilon^{2}\right) .
$$

This proves the statement of the theorem.

An analogous expansion for $C^{2}$ functions in terms of surface integrals can also be derived. Its precise expression is given in the statement of Proposition 3.2 below.

Proposition 3.2. Let $\Omega \subset \mathcal{R}^{2}$ be a smooth domain. Given $\phi \in C^{2}(\Omega)$ and $x_{0} \in \Omega$ for which $\nabla \phi\left(x_{0}\right) \neq 0$, we have that for any $\epsilon>0$ such that $B_{\epsilon}\left(x_{0}\right) \subset \Omega$ it holds that

$$
\phi\left(x_{0}\right)=\frac{\int_{\partial B_{\epsilon}\left(x_{0}\right)}\left|\nabla \phi\left(x_{0}\right) \cdot\left(x-x_{0}\right)\right|^{p-2} \phi(x) d x}{\int_{\partial B_{\epsilon}\left(x_{0}\right)}\left|\nabla \phi\left(x_{0}\right) \cdot\left(x-x_{0}\right)\right|^{p-2} d x}-\frac{\epsilon^{2}}{2} \Delta_{p}^{G} \phi\left(x_{0}\right)+o\left(\epsilon^{2}\right) .
$$


Proof. Equation (17) is obtained by following the proof of Theorem 3.1 step by step, with straightforward modifications.

At a point $x_{0}$ where the gradient of the function is zero, when considering the Game $p$-Laplacian in Section 5 we will use the following classical formulas (in the spirit of the works [2, 8] ), which are obtained by integrating over $B_{\epsilon}\left(x_{0}\right)$ and $\partial B_{\epsilon}\left(x_{0}\right)$ the Taylor expansion.

Lemma 3.3. Let $\phi \in C^{2}(\Omega) \subset \mathcal{R}^{2}$. At each point $x_{0}$ where $\nabla \phi\left(x_{0}\right)=0$, given any $\epsilon>0$ such that $B_{\epsilon}\left(x_{0}\right) \subset \Omega$, it holds that

$$
\phi\left(x_{0}\right)=\frac{1}{\left|B_{\epsilon}\left(x_{0}\right)\right|} \int_{B_{\epsilon}\left(x_{0}\right)} \phi(x) d x-\frac{\epsilon^{2}}{4} \Delta_{2}^{G} \phi\left(x_{0}\right)+o\left(\epsilon^{2}\right)
$$

and

$$
\phi\left(x_{0}\right)=\frac{1}{\left|\partial B_{\epsilon}\left(x_{0}\right)\right|} \int_{\partial B_{\epsilon}\left(x_{0}\right)} \phi(x) d x-\frac{\epsilon^{2}}{2} \Delta_{2}^{G} \phi\left(x_{0}\right)+o\left(\epsilon^{2}\right) .
$$

\section{WEAK MEAN VALUe PROPERTy}

Manfredi et al. in [6] introduce a definition of asymptotic equality in the viscosity sense, which we use to specify in which sense we claim that a $p$-harmonic function verifies a mean value property.

Definition 4.1. Let $\Omega \subset \mathcal{R}^{2}$ be a smooth domain, and let $x_{0} \in \Omega$. We say that $u \in C^{0}(\Omega)$ verifies the equality

$$
u\left(x_{0}\right)=\frac{\int_{B_{\epsilon}\left(x_{0}\right)}\left|\nabla u\left(x_{0}\right) \cdot\left(x-x_{0}\right)\right|^{p-2} u(x) d x}{\int_{B_{\epsilon}\left(x_{0}\right)}\left|\nabla u\left(x_{0}\right) \cdot\left(x-x_{0}\right)\right|^{p-2} d x}+o\left(\epsilon^{2}\right),
$$

in the viscosity sense, if the following conditions hold:

(i) For any $\phi \in C^{2}$ for which $u-\phi$ has a strict local maximum at $x_{0} \in \Omega$, there exists an $\epsilon_{0}>0$ such that for every $\epsilon<\epsilon_{0}$,

$$
\phi\left(x_{0}\right) \leq \frac{\int_{B_{\epsilon}\left(x_{0}\right)}\left|\nabla \phi\left(x_{0}\right) \cdot\left(x-x_{0}\right)\right|^{p-2} \phi(x) d x}{\int_{B_{\epsilon}\left(x_{0}\right)}\left|\nabla \phi\left(x_{0}\right) \cdot\left(x-x_{0}\right)\right|^{p-2} d x}+o\left(\epsilon^{2}\right),
$$

whenever $\nabla \phi(x) \neq 0$.

(ii) For any $\phi \in C^{2}$ for which $u-\phi$ has a strict local minimum at $x_{0} \in \Omega$, there exists an $\epsilon_{0}>0$ such that for every $\epsilon<\epsilon_{0}$,

$$
\phi\left(x_{0}\right) \geq \frac{\int_{B_{\epsilon}\left(x_{0}\right)}\left|\nabla \phi\left(x_{0}\right) \cdot\left(x-x_{0}\right)\right|^{p-2} \phi(x) d x}{\int_{B_{\epsilon}\left(x_{0}\right)}\left|\nabla \phi\left(x_{0}\right) \cdot\left(x-x_{0}\right)\right|^{p-2} d x}+o\left(\epsilon^{2}\right),
$$

whenever $\nabla \phi(x) \neq 0$.

We are now ready to prove our representation formula.

Theorem 4.1. Let $u$ be a continuous function in $\Omega \subset \mathcal{R}^{2}$, and let $x_{0} \in \Omega$. For any $\epsilon>0$ such that $B_{\epsilon}\left(x_{0}\right) \subset \Omega$ we have that

$$
u\left(x_{0}\right)=\frac{\int_{B_{\epsilon}\left(x_{0}\right)}\left|\nabla u\left(x_{0}\right) \cdot\left(x-x_{0}\right)\right|^{p-2} u(x) d x}{\int_{B_{\epsilon}\left(x_{0}\right)}\left|\nabla u\left(x_{0}\right) \cdot\left(x-x_{0}\right)\right|^{p-2} d x}+o\left(\epsilon^{2}\right)
$$


holds in the viscosity sense if and only if $u$ is p-harmonic, that is, if $u$ is a viscosity solution of

$$
\Delta_{p} u\left(x_{0}\right)=0 .
$$

Proof. Assume (20) holds; we need to show that $u$ is $p$-harmonic in $\Omega$. We will show that $u$ is a subsolution of (21) according to Definition 2.1, the proof that $u$ is a supersolution is similar.

Let $\phi$ be such that $u-\phi$ has a strict local maximum at $x_{0} \in \Omega$, and assume that $\nabla \phi\left(x_{0}\right) \neq 0$. Then by equation (18) in Definition 4.1 (18) there exists an $\epsilon_{0}>0$ such that for every $\epsilon<\epsilon_{0}$,

$$
\phi\left(x_{0}\right) \leq \frac{\int_{B_{\epsilon}\left(x_{0}\right)}\left|\nabla \phi\left(x_{0}\right) \cdot\left(x-x_{0}\right)\right|^{p-2} \phi(x) d x}{\int_{B_{\epsilon}\left(x_{0}\right)}\left|\nabla \phi\left(x_{0}\right) \cdot\left(x-x_{0}\right)\right|^{p-2} d x}+o\left(\epsilon^{2}\right) .
$$

On the other hand, since $\phi \in C^{2}$ by Theorem 3.1, as long as $B_{\epsilon}\left(x_{0}\right) \subset \Omega$ we have

$$
\phi\left(x_{0}\right)=\frac{\int_{B_{\epsilon}\left(x_{0}\right)}\left|\nabla \phi\left(x_{0}\right) \cdot\left(x-x_{0}\right)\right|^{p-2} \phi(x) d x}{\int_{B_{\epsilon}\left(x_{0}\right)}\left|\nabla \phi\left(x_{0}\right) \cdot\left(x-x_{0}\right)\right|^{p-2} d x}-\frac{p}{p+2} \frac{\epsilon^{2}}{2} \Delta_{p}^{G} \phi\left(x_{0}\right)+o\left(\epsilon^{2}\right) .
$$

Thus for $\epsilon<\epsilon_{0}$ it holds that

$$
-\frac{p}{p+2} \frac{\epsilon^{2}}{2} \Delta_{p}^{G} \phi\left(x_{0}\right)+o\left(\epsilon^{2}\right) \leq o\left(\epsilon^{2}\right)
$$

an inequality which we divide by $\epsilon^{2}$ and let $\epsilon$ go to zero to obtain

$$
-\Delta_{p}^{G} \phi\left(x_{0}\right) \leq 0
$$

Finally, since $\phi$ is a $C^{2}$ function, we can use (4) and (3) to conclude that

$$
0 \geq-\Delta_{p}^{G} \phi=-\frac{1}{p}\left(\Delta_{2} \phi-\Delta_{\infty}^{G} \phi\right)-\frac{1}{q} \Delta_{\infty}^{G} \phi=-\frac{1}{p} \Delta_{2} \phi-\frac{p-2}{p} \Delta_{\infty}^{G} \phi,
$$

which gives that $u$ is a subsolution of $\Delta_{p} u\left(x_{0}\right)=0$.

Assume next that $u$ is a viscosity supersolution. We are going to show that (19) holds.

Let $\phi \in C^{2}$ be such that $u-\phi$ has a strict local minimum at $x_{0} \in \Omega$ and $\nabla \phi\left(x_{0}\right) \neq 0$. By (8) it follows that

$$
-(p-2) \Delta_{\infty}^{G} \phi\left(x_{0}\right)-\Delta_{2} \phi\left(x_{0}\right) \geq 0,
$$

which as in (22) gives $-\Delta_{p}^{G} \phi\left(x_{0}\right) \geq 0$. Hence, by Theorem 3.1 for any $\epsilon<\epsilon_{0}$, where $\epsilon_{0}$ is picked so that $B_{\epsilon_{0}}\left(x_{0}\right) \subset \Omega$, we obtain

$$
\phi\left(x_{0}\right) \geq \frac{\int_{B_{\epsilon}\left(x_{0}\right)}\left|\nabla \phi\left(x_{0}\right) \cdot\left(x-x_{0}\right)\right|^{p-2} \phi(x) d x}{\int_{B_{\epsilon}\left(x_{0}\right)}\left|\nabla \phi\left(x_{0}\right) \cdot\left(x-x_{0}\right)\right|^{p-2} d x}+o\left(\epsilon^{2}\right),
$$

which is exactly (19).

In a similar way, if $u$ is a subsolution, one can show that (18) holds, which proves that if $u$ is $p$-harmonic, then it verifies (20) in the viscosity sense. This concludes the proof of our theorem.

With the due modifications in Definition 4.1, one can also derive a representation for $p$-harmonic functions which uses only the values on the surface of the ball. 
Theorem 4.2. Let $u$ be a continuous function in $\Omega \subset \mathcal{R}^{2}$, and let $x_{0} \in \Omega$. For any $\epsilon>0$ such that $B_{\epsilon}\left(x_{0}\right) \subset \Omega$ we have that

$$
u\left(x_{0}\right)=\frac{\int_{\partial B_{\epsilon}\left(x_{0}\right)}\left|\nabla u\left(x_{0}\right) \cdot\left(x-x_{0}\right)\right|^{p-2} u(x) d x}{\int_{\partial B_{\epsilon}\left(x_{0}\right)}\left|\nabla u\left(x_{0}\right) \cdot\left(x-x_{0}\right)\right|^{p-2} d x}+o\left(\epsilon^{2}\right)
$$

holds in the viscosity sense if and only if $u$ is p-harmonic, that is, if $u$ is a viscosity solution of

$$
\Delta_{p} u\left(x_{0}\right)=0 .
$$

Proof. The result follows as in the proof of Theorem 4.1, thanks to Proposition 3.2

\section{Representation for the Game $p$-Laplacian}

The definition of asymptotic equality in the viscosity sense for the case of the Game $p$-Laplacian needs to be adapted to a different definition of viscosity solution since one needs to account for the fact the the operator is singular.

Definition 5.1. Let $\Omega \subset \mathcal{R}^{2}$ be a smooth domain, and let $x_{0} \in \Omega$. If $f$ is a continuous function, we say that $u \in C^{0}(\Omega)$ verifies the equality

$$
u\left(x_{0}\right)=\frac{\int_{B_{\epsilon}\left(x_{0}\right)}\left|\nabla u\left(x_{0}\right) \cdot\left(x-x_{0}\right)\right|^{p-2} u(x) d x}{\int_{B_{\epsilon}\left(x_{0}\right)}\left|\nabla u\left(x_{0}\right) \cdot\left(x-x_{0}\right)\right|^{p-2} d x}+\frac{\epsilon^{2}}{2} \frac{p}{p+2} f\left(x_{0}\right)+o\left(\epsilon^{2}\right),
$$

in the viscosity sense, if the following conditions hold:

(i) For any $\phi \in C^{2}(\Omega)$ for which $u-\phi$ has a local maximum at $x_{0} \in \Omega$, there exists an $\epsilon_{0}>0$ such that for every $\epsilon<\epsilon_{0}$,

$$
\phi\left(x_{0}\right) \leq \frac{\int_{B_{\epsilon}\left(x_{0}\right)}\left|\nabla \phi\left(x_{0}\right) \cdot\left(x-x_{0}\right)\right|^{p-2} \phi(x) d x}{\int_{B_{\epsilon}\left(x_{0}\right)}\left|\nabla \phi\left(x_{0}\right) \cdot\left(x-x_{0}\right)\right|^{p-2} d x}+\frac{\epsilon^{2}}{2} \frac{p}{p+2} f\left(x_{0}\right)+o\left(\epsilon^{2}\right),
$$

whenever $\nabla \phi(x) \neq 0$; and

$$
\phi\left(x_{0}\right) \leq \frac{1}{\left|B_{\epsilon}\left(x_{0}\right)\right|} \int_{B_{\epsilon}\left(x_{0}\right)} \phi(x) d x+\frac{\epsilon^{2}}{4} f\left(x_{0}\right)+o\left(\epsilon^{2}\right), \text { whenever } \nabla \phi(x)=0 .
$$

(ii) For any $\phi \in C^{2}(\Omega)$ for which $u-\phi$ has a local minimum at $x_{0} \in \Omega$, there exists an $\epsilon_{0}>0$ such that for every $\epsilon<\epsilon_{0}$,

$$
\phi\left(x_{0}\right) \geq \frac{\int_{B_{\epsilon}\left(x_{0}\right)}\left|\nabla \phi\left(x_{0}\right) \cdot\left(x-x_{0}\right)\right|^{p-2} \phi(x) d x}{\int_{B_{\epsilon}\left(x_{0}\right)}\left|\nabla \phi\left(x_{0}\right) \cdot\left(x-x_{0}\right)\right|^{p-2} d x}+\frac{\epsilon^{2}}{2} \frac{p}{p+2} f\left(x_{0}\right)+o\left(\epsilon^{2}\right),
$$

whenever $\nabla \phi(x) \neq 0$; and

$$
\phi\left(x_{0}\right) \geq \frac{1}{\left|B_{\epsilon}\left(x_{0}\right)\right|} \int_{B_{\epsilon}\left(x_{0}\right)} \phi(x) d x+\frac{\epsilon^{2}}{4} f\left(x_{0}\right)+o\left(\epsilon^{2}\right), \text { whenever } \nabla \phi(x)=0 .
$$

We can then prove the following result.

Theorem 5.1. Let $f$ and $u$ be continuous functions in $\Omega \subset \mathcal{R}^{2}$, and let $x_{0} \in \Omega$. We have that

$$
u\left(x_{0}\right)=\frac{\int_{B_{\epsilon}\left(x_{0}\right)}\left|\nabla u\left(x_{0}\right) \cdot\left(x-x_{0}\right)\right|^{p-2} u(x) d x}{\int_{B_{\epsilon}\left(x_{0}\right)}\left|\nabla u\left(x_{0}\right) \cdot\left(x-x_{0}\right)\right|^{p-2} d x}+\frac{\epsilon^{2}}{2} \frac{p}{p+2} f\left(x_{0}\right)+o\left(\epsilon^{2}\right)
$$


holds in the viscosity sense in $\Omega$ if and only if $u$ is a viscosity solution of

$$
-\Delta_{p}^{G} u\left(x_{0}\right)=f\left(x_{0}\right) \text { in } \Omega \text {. }
$$

Proof. Assume that (27) holds; we need to show that $u$ is a viscosity solution. Recalling Definition 2.3 of viscosity solution it should be clear that part (i) of the definition of a subsolution can be obtained with almost the same proof as in Theorem 4.1. If instead $\phi \in C^{2}(\Omega)$ is such that $u-\phi$ has a local maximum at $x_{0} \in \Omega$ and $\nabla \phi\left(x_{0}\right)=0$, by (24) we know there exists an $\epsilon_{0}>0$ such that for every $\epsilon<\epsilon_{0}$,

$$
\phi\left(x_{0}\right) \leq \frac{1}{\left|B_{\epsilon}\left(x_{0}\right)\right|} \int_{B_{\epsilon}\left(x_{0}\right)} \phi(x) d x+\frac{\epsilon^{2}}{4} f\left(x_{0}\right)+o\left(\epsilon^{2}\right),
$$

and by Lemma 3.3. we conclude as in Theorem 4.1 that

$$
-\Delta_{2}^{G} \phi\left(x_{0}\right) \leq f\left(x_{0}\right) \text {. }
$$

The case $u$ supersolution is analogous.

Assume next that $u$ is a viscosity supersolution of $-\Delta_{p}^{G} \phi=f$ in $\Omega$. We need to show that (25) and (26) are satisfied.

Again (25) follows as in Theorem 4.1 assume then that $\phi \in C^{2}(\Omega)$ is such that $u-\phi$ has a local minimum at $x_{0} \in \Omega$ and $\nabla \phi\left(x_{0}\right)=0$. By part (ii)' of the definition of a supersolution this implies

$$
-\Delta_{2}^{G} \phi\left(x_{0}\right) \geq f\left(x_{0}\right)
$$

but by Lemma 3.3 for any $\epsilon<\epsilon_{0}$, where $\epsilon_{0}$ is picked so that $B_{\epsilon_{0}}\left(x_{0}\right) \subset \Omega$, we derive

$$
\phi\left(x_{0}\right) \geq \frac{\int_{B_{\epsilon}\left(x_{0}\right)}\left|\nabla \phi\left(x_{0}\right) \cdot\left(x-x_{0}\right)\right|^{p-2} \phi(x) d x}{\int_{B_{\epsilon}\left(x_{0}\right)}\left|\nabla \phi\left(x_{0}\right) \cdot\left(x-x_{0}\right)\right|^{p-2} d x}+\frac{\epsilon^{2}}{4} f\left(x_{0}\right)+o\left(\epsilon^{2}\right),
$$

which is exactly (25).

In a similar fashion, if $u$ is a subsolution one can prove that (23) and (24) hold.

We conclude this section with the corresponding theorem with surface integrals, similar to what was done in Theorem 4.2 for $p$-harmonic functions. We start by giving the appropriate definition of asymptotic equality in the viscosity sense.

Definition 5.2. Let $\Omega \subset \mathcal{R}^{2}$ be a smooth domain, and let $x_{0} \in \Omega$. If $f$ is a continuous function, we say that $u \in C^{0}(\Omega)$ verifies the equality

$$
u\left(x_{0}\right)=\frac{\int_{\partial B_{\epsilon}\left(x_{0}\right)}\left|\nabla u\left(x_{0}\right) \cdot\left(x-x_{0}\right)\right|^{p-2} u(x) d x}{\int_{\partial B_{\epsilon}\left(x_{0}\right)}\left|\nabla u\left(x_{0}\right) \cdot\left(x-x_{0}\right)\right|^{p-2} d x}+\frac{\epsilon^{2}}{2} f\left(x_{0}\right)+o\left(\epsilon^{2}\right),
$$

in the viscosity sense, if the following conditions hold:

(i) For any $\phi \in C^{2}(\Omega)$ for which $u-\phi$ has a local maximum at $x_{0} \in \Omega$, there exists an $\epsilon_{0}>0$ such that for every $\epsilon<\epsilon_{0}$,

$$
\phi\left(x_{0}\right) \leq \frac{\int_{\partial B_{\epsilon}\left(x_{0}\right)}\left|\nabla \phi\left(x_{0}\right) \cdot\left(x-x_{0}\right)\right|^{p-2} \phi(x) d x}{\int_{\partial B_{\epsilon}\left(x_{0}\right)}\left|\nabla \phi\left(x_{0}\right) \cdot\left(x-x_{0}\right)\right|^{p-2} d x}+\frac{\epsilon^{2}}{2} f\left(x_{0}\right)+o\left(\epsilon^{2}\right),
$$

whenever $\nabla \phi(x) \neq 0$; and

$$
\phi\left(x_{0}\right) \leq \frac{1}{\left|\partial B_{\epsilon}\left(x_{0}\right)\right|} \int_{\partial B_{\epsilon}\left(x_{0}\right)} \phi(x) d x+\frac{\epsilon^{2}}{2} f\left(x_{0}\right)+o\left(\epsilon^{2}\right), \text { whenever } \nabla \phi(x)=0 .
$$


(ii) For any $\phi \in C^{2}(\Omega)$ for which $u-\phi$ has a local minimum at $x_{0} \in \Omega$, there exists an $\epsilon_{0}>0$ such that for every $\epsilon<\epsilon_{0}$,

$$
\phi\left(x_{0}\right) \geq \frac{\int_{\partial B_{\epsilon}\left(x_{0}\right)}\left|\nabla \phi\left(x_{0}\right) \cdot\left(x-x_{0}\right)\right|^{p-2} \phi(x) d x}{\int_{\partial B_{\epsilon}\left(x_{0}\right)}\left|\nabla \phi\left(x_{0}\right) \cdot\left(x-x_{0}\right)\right|^{p-2} d x}+\frac{\epsilon^{2}}{2} f\left(x_{0}\right)+o\left(\epsilon^{2}\right),
$$

whenever $\nabla \phi(x) \neq 0$; and

$$
\phi\left(x_{0}\right) \geq \frac{1}{\left|\partial B_{\epsilon}\left(x_{0}\right)\right|} \int_{\partial B_{\epsilon}\left(x_{0}\right)} \phi(x) d x+\frac{\epsilon^{2}}{2} f\left(x_{0}\right)+o\left(\epsilon^{2}\right) \text {, whenever } \nabla \phi(x)=0 .
$$

We leave to the reader the proof of the last theorem, since it is a simple modification of the proof of Theorem 5.1 above.

Theorem 5.2. Let $f$ and $u$ be continuous functions in $\Omega \subset \mathcal{R}^{2}$, and let $x_{0} \in \Omega$. We have that

$$
u\left(x_{0}\right)=\frac{\int_{\partial B_{\epsilon}\left(x_{0}\right)}\left|\nabla u\left(x_{0}\right) \cdot\left(x-x_{0}\right)\right|^{p-2} u(x) d x}{\int_{\partial B_{\epsilon}\left(x_{0}\right)}\left|\nabla u\left(x_{0}\right) \cdot\left(x-x_{0}\right)\right|^{p-2} d x}+\frac{\epsilon^{2}}{2} f\left(x_{0}\right)+o\left(\epsilon^{2}\right)
$$

holds in the viscosity sense in $\Omega$ if and only if $u$ is a viscosity solution of

$$
-\Delta_{p}^{G} u\left(x_{0}\right)=f\left(x_{0}\right) \text { in } \Omega \text {. }
$$

\section{REFERENCES}

[1] G. Barles and P.E. Souganidis. Convergence of approximation schemes for fully nonlinear second order equations. Asymptotic Anal. 4 (1991), 271-283. MR.1115933 (92d:35137)

[2] W Blaschke. Ein Mittelwertsatz und eine kennzeichnende Eigenschaft des logarithmischen Potentials. Leipz. Ber. 68 (1916), 37.

[3] M. G. Crandall, H. Ishii and P.-L. Lions. User's guide to viscosity solutions of second order partial differential equations. Bull. Amer. Math. Soc. (N.S.) 27 (1992), no. 1, 1-67. MR1118699 $(92 \mathrm{j}: 35050)$

[4] M. Falcone, S. Finzi Vita, T. Giorgi and R. Smits. A semi-Lagrangian scheme for the Game $p$-Laplacian via $p$-averaging. Submitted.

[5] P. Juutinen, P. Lindqvist and J. Manfredi. On the equivalence of viscosity solutions and weak solutions for a quasi-linear equation. SIAM J. Math. Anal. 33 (2001), no. 3, 699-717. MR:1871417(2002m:35051)

[6] J. Manfredi, M. Parviainen and J. D. Rossi. An asymptotic mean value characterization for p-harmonic functions. Proc. Amer. Math. Soc. 138 (2010), no. 3, 881-889. MR2566554 (2010k:35200)

[7] Y. Peres and S. Sheffield. Tug-of-war with noise: a game-theoretic view of the $p$-Laplacian. Duke Math. J. 145 (2008), 91-120. MR2451291(2010i:35100)

[8] I. Privaloff. Sur les fonctions harmoniques. Rec. Math. Moscou (Mat. Sbornik) 32 (1925), 464-471.

[9] P. Wang. A formula for smooth $\infty$-harmonic functions. PanAmerican Mathematical Journal 16 (2006), no. 1, 57-65. MR2186538

Department of Mathematical Sciences, New Mexico State University, Las Cruces, New Mexico 88003-8001

E-mail address: tgiorgi@nmsu.edu

Department of Mathematical Sciences, New Mexico State University, Las Cruces, NeW MeXico 88003-8001

E-mail address: rsmits@nmsu.edu 